\title{
Neumoperitoneo en paciente con tratamiento renal sustitutivo con diálisis peritoneal continua ambulatoria
}

\author{
María Isabel Durán Muñoz*, Consuelo Martín Santiago*, Piedad de la Cierva Medina*, Teresa Lope An-

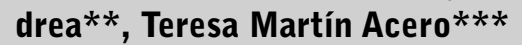 \\ *Enfermeras, * * Supervisora del Servicio de Diálisis, * * Subdirectora de Enfermería del Hospital Clínico San \\ Carlos. Madrid
}

\section{Introducción:}

Se denomina neumoperitoneo a la presencia de aire en la cavidad peritoneal. La mayoría de las veces, $85-95 \%$ de las ocasiones, la causa del neumoperitoneo es la perforación de una víscera hueca, pero el $5-15 \%$ de los casos son no quirúrgicos y se definen como neumoperitoneo no quirúrgico, asintomático, benigno o idiopático, que se resuelven con un tratamiento conservador.

A continuación describimos el caso clínico de una paciente que presentó un neumoperitoneo.

\section{Caso clínico:}

Mujer de 73 años de edad con antecedentes personales de cardiopatía hipertensiva, hipercolesterolemia, diabetes tipo II, histerectomía por prolapso uterino y corrección de cistocele, hipotiroidismo primario, neuralgia del trigémino, asma bronquial, depresión, arterioesclerosis, colecistectomía por adenomioma de vesícula, insuficiencia suprarrenal y nefrectomía bilateral: derecha por pseudotumoración e izquierda por hematoma abcesificado, lo que la lleva a permanecer en tratamiento renal sustitutivo con diálisis peritoneal continua ambulatoria (DPCA) desde Enero del 2011. Durante el periodo de entrenamiento y en consultas posteriores, no se evidenció una mala realización de la técnica por parte de la paciente, ni refirió síntomas de neumoperitoneo.

En Marzo del 2013, acude a urgencias por un cuadro de una semana de evolución de dolor abdominal, diarrea, nauseas sin vómitos y escalofríos sin fiebre.

\footnotetext{
María Isabel Durán Muñoz

Profesor Martín Lagos s/n 28040 Madrid

E-mail: iduran72@gmail.com
}

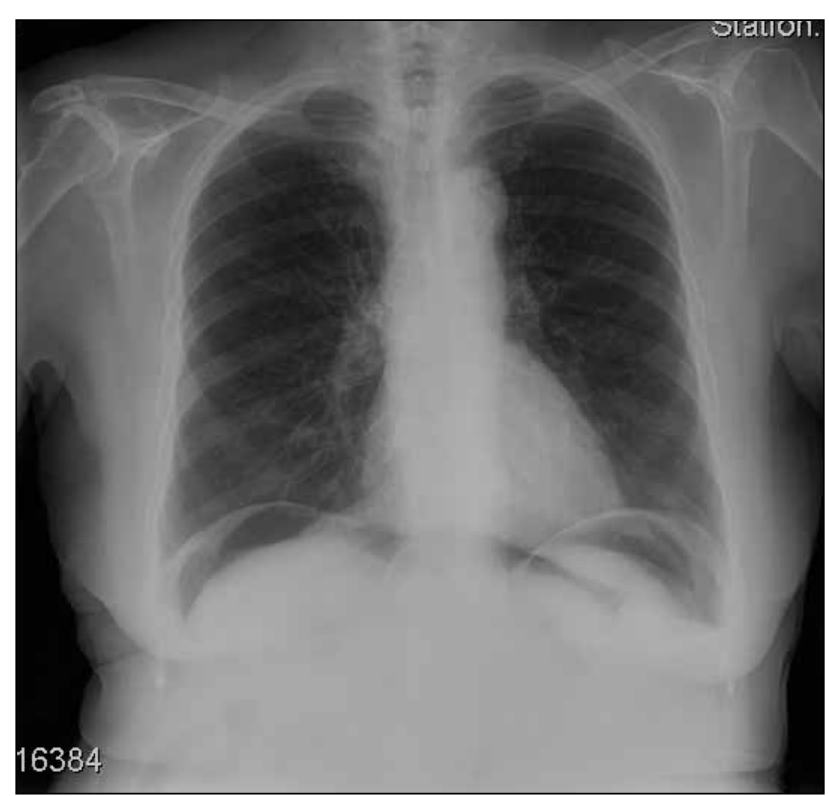

Figura 1. Radiografía de tórax diagnóstica.

En la exploración física de urgencias presenta dolor a la palpación profunda de predominio en fosa iliaca derecha, palidez cutánea y extremidades sin edemas. TA: 100/60, Fc: 94 Ipm., afebril, Fr: 24 rpm, Sat.02:89\%.

Se realizó analítica (Hemograma, bioquímica y gasometría venosa), radiografía de tórax (Figura 1) y abdomen donde aparece catéter de diálisis peritoneal bien colocado y neumoperitoneo. Tras ser valorada por el servicio de cirugía, se realiza TAC con contraste oral (Figura 2) donde aparece un neumoperitoneo importante, sin apreciarse fugas del contraste ni perforación de una víscera hueca.

Una vez realizada la valoración inicial de enfermería, con los datos anteriores y la entrevista a la paciente, no se aprecian problemas con la realización de la técnica.

Procedemos a realizar intercambio de diálisis peritoneal según protocolo, para valorar la posibilidad de in- 


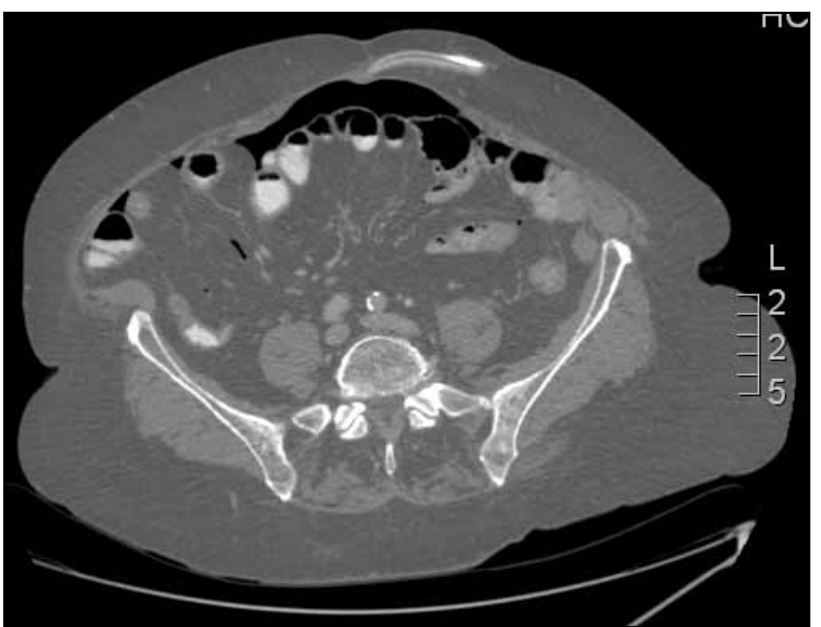

Figura 2. TAC diagnóstico de abdomen con contraste oral.

fección peritoneal. No se evidencian signos de infección (Líquido peritoneal claro), se recoge muestra de líquido para cultivo y análisis, con resultado de: leucocitos: $80 /$ $\mathrm{uL}$, descartando así la infección peritoneal.

Dada la situación de la paciente, se procede al ingreso para observación. Se deja en dieta absoluta y con sueroterapia.

Ante la posibilidad de que el neumoperitoneo esté en relación con la técnica de diálisis peritoneal, se decide que el personal de enfermería realice los intercambios de diálisis peritoneal según pauta y tras el drenaje, colocar a la paciente en posición de Trendelemburg para favorecer la salida del aire peritoneal.

En estudios radiológicos posteriores se observa la disminución paulatina del neumoperitoneo, hasta quedar resuelto casi en su totalidad al alta de la paciente. (Figura 3).

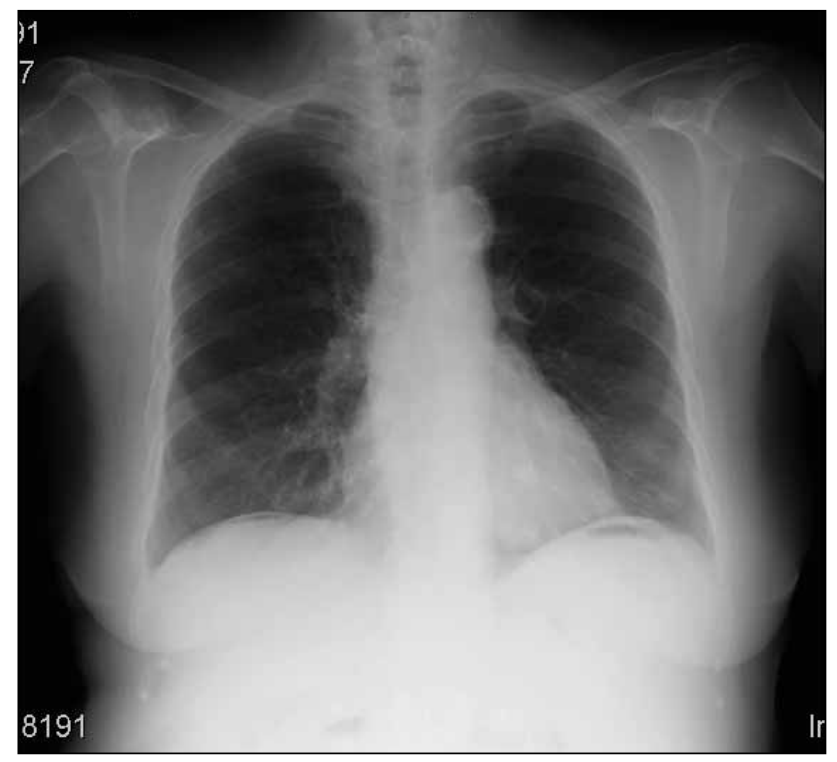

Figura 3. Radiografía de Tórax al alta.

\section{Conclusión:}

Ante la presencia del neumoperitoneo, la primera actitud estaba encaminada a la sospecha de la perforación de una víscera hueca y, por tanto, la práctica de una laparotomía urgente. Sin embargo, los antecedentes patológicos, la historia clínica, la exploración física y los análisis complementarios eran favorables a optar por un manejo no quirúrgico, por la estrecha vigilancia de la paciente y ante cualquier empeoramiento, practicar la laparotomía.

Creemos, que el neumoperitoneo que apareció en esta paciente, fue causado por una mala realización en el purgado del sistema de diálisis peritoneal durante la realización de este tratamiento en su domicilio.

En este caso, la actuación del personal de enfermería fue primordial para la resolución del problema de la paciente, dado que, fue la enfermera, la que realizó los intercambios de DPCA mientras la paciente estuvo ingresada en nuestro hospital y la que llevó a cabo dicha técnica, pudiendo verificar la salida del aire del peritoneo a la bolsa de drenaje cada vez que se realizaban los intercambios de DPCA.

Una vez resuelto el neumoperitoneo, el personal de enfermería, llevó a cabo un nuevo re-entrenamiento de la paciente, para reforzar la técnica de DPCA que debía seguir en su domicilio, haciendo hincapié en la fase de purgado del sistema.

Hemos de decir, que hasta la fecha de hoy, no ha aparecido un nuevo episodio de neumoperitoneo en dicha paciente, lo que nos hace pensar que la educación sanitaria recibida por parte del personal de enfermería, ha sido la adecuada.

Recibido: 4 Junio 2013

Revisado: 20 Junio 2013

Modificado: 25 Junio 2013

Aceptado: 1 Julio 2013 


\section{Bibliografía:}

1. López-Cano, Manuel; Vilallonga-Puy, Ramón; Lozoya-Trujillo, Roberto; Espin-Basany, Eloi; Luis Sánchez-García, José; Armengol-Carrasco, Manel. Neumoperitoneo idiopático. Publicado en Cir. Esp. 2005; 78:112-4. - vol.78 núm. 02.

2. Martínez Villena, Beatriz; Ishaq Humaid, Wael; Gómez Marco, José Javier. Neumoperitoneo espontáneo. Published in FMC. Form Med Contin Aten Prim. 2007; Vol.14 núm. 09 14:576-7.
3. García González Carlos Alejandro, Castro Carvajal Juan Camilo. Neumoperitoneo espontaneo: Presentación de caso. Rev. Colomb Radiol 2012; 23(1): 3446-3448.

4. Fernández Arroyo Lucila, Martínez Alcon Berta, Grande Velasco Julita, García Lorenzo Ana Isabel. Dos casos de neumoperitoneo en pacientes en DPCA asociados al uso de solución Bicavera ${ }^{\circledR}$. Libro de comunicaciones del XXXV Congreso de la sociedad Española de Enfermería Nefrológica.2010; Pág. 300. 